\title{
Is allogeneic transplantation the preferred therapy for older patients with acute myeloid leukemia?
}

Arnold Ganser

Department of Hematology, Hemostasis, Oncology, and Stem Cell Transplantation, Hannover Medical School, Hannover, Germany

E-mail: ganser.arnold@mh-hannover.de

https://doi.org/10.3324/haematol.2021.279820

The treatment of acute myeloid leukemia (AML) has seen tremendous developments over the last few years, but treatment approaches and results are predominantly determined by selection based on age, karyotype, and molecular genotype. Treatment recommendations mainly follow the European LeukemiaNet guidelines, especially regarding the use of allogeneic hematopoietic stem cell transplantation (HSCT) as post-remission consolidation therapy for adverse-risk disease. However, these recommendations are category $2 \mathrm{~A}$ only with low-level evidence but uniform panel consensus, since results from randomized clinical trials are lacking. ${ }^{2}$ The deficits with the system, in addition, include a bias in that the dataset for its establishment almost exclusively consists of treatment results in younger fit patients with de novo AML who were treated with intensive induction chemotherapy followed by high-dose cytosine arabinoside or HSCT. Recommendations for post-remission therapy in elderly AML patients have not explicitly addressed HSCT but point to the high degree of selection even with reduced intensity conditioning $(\mathrm{RIC}) .^{3}$

In this issue of Haematologica, Russell et al. now present a considerable set of data on the outcome of older AML patients, aged 60 to 70 years, treated intensively within the National Cancer Research Institute (NCRI) AML 16 study with various induction regimens followed by RIC transplantation in the case of non-favorable cytogenetics and the presence of a fully matched related or unrelated (at least 9/10 HLA-match) donor. ${ }^{4}$ Out of 932 patients treated between 2006 and 2012, 788 continued on some sort of chemotherapy, while 144 underwent HSCT (sibling $n=52$; matched unrelated donor $n=92$ ). The survival rate at 5 years was $37 \%$ among the transplanted patients compared to $20 \%$ in the chemotherapy arm $(P<0.001)$. There was no significant difference in survival between patients transplanted with grafts from siblings or matched unrelated donors. Dividing patients into groups according to Wheatley risk, ${ }^{5}$ all three risk groups benefited from HSCT. Although mutation status was not known in the majority of patients, benefit was also seen in patients with an FLT3-ITD and/or NPM1 mutation with no difference among genotypic subgroups. Thus, Russell et al. conclude that "RIC transplantation is an attractive option for older AML patients lacking favorable risk cytogenetics". ${ }^{4}$

Although the data are encouraging they do not fully solve the current problems in the elderly AML patient population with regard to appropriate post-remission therapy. In the NRCI AML 16 trial the patients had to be fit for intensive induction chemotherapy which only applies to the minority of the patients. Recent developments with new effective drugs and combination therapies have not yet been addressed. These include, for example, the use of additional FLT3 inhibitors ${ }^{6}$ or CPX- $351^{7}$ in fit patients who can be treated intensively, or combinations of hypomethylating agents plus venetoclax which have now become standard of care in less fit AML patients. The latter combination is especially effective in AML with NPM1 and IDH mutations. ${ }^{8}$ These developments, especially in the unfit population, might even lead to a more dynamic approach, since patients unfit at the time of AML diagnosis might become fit for RIC HSCT once they have entered complete remission with restoration of normal hematopoietic function. ${ }^{9}$ The NCRI AML 16 data support the now common practice of offering RIC HSCT as post-remission therapy to these patients. However, it would be preferable to design randomized controlled trials to demonstrate the advantage of this approach compared to other post-remission therapies including maintenance therapy, e.g., with oral azacitidine. ${ }^{10}$ The development of novel drugs will certainly lead to new risk stratification in $A M L$ and treatment recommendations which include HSCT also in older AML patients. ${ }^{11}$ Not only has HSCT undergone rapid progress, but the whole therapeutic landscape is in flux and it would be desirable to have data from randomized controlled trials in order to inform decisions in patients' care.

\section{Disclosures}

No conflicts of interest to disclose.

\section{References}

1. Döhner H, Estey E, Grimwade D, et al. Diagnosis and management of AML in adults: 2017 ELN recommendations from an international expert panel. Blood. 2017;129(4):424-447.
2. Kim MS, Cai J, Maniar A, et al. Comparison of classification of indications for allogeneic and autologous transplant for adults in ASTCT guidelines and evidence available in published 
literature. JAMA Intern Med. 2021;e214826.

3. Sekeres MA, Guyatt G, Abel G, et al. American Society of Hematology 2020 guidelines for treating newly diagnosed acute myeloid leukemia in older adults. Blood Adv. 2020;4(15):3528-3549.

4. Russell NH, Hills RK, Thoma A, et al. Outcomes of older patients aged 60 to 70 years undergoing reduced intensity transplant for acute myeloblastic leukemia: results of the NCRI acute myeloid leukemia 16 trial. Haematologica. 2022;107(7):1518-1527.

5. Wheatley K, Brookes CL, Howman AJ, et al. Prognostic factor analysis of the survival of elderly patients with $A M L$ in the MRC AML 11 and LRF AML 14 trials. Br J Haematol. 2009;145(5):598-605.

6. Stone RM, Mandrekar SJ, Sanford BL, et al. Midostaurin plus chemotherapy for acute myeloid leukemia with a FLT3 mutation. N Engl J Med. 2017;377(5):454-464.

7. Lancet JE, Uy GL, Cortes JE, et al. CPX-351 (cytarabine and daunorubicin) liposome for injection versus conventional cytarabine plus daunorubicin in older patients with newly diagnosed secondary acute myeloid leukemia. J Clin Oncol. 2018;36(26):2684-2692.

8. DiNardo CD, Jonas BA, Pullarkat V, et al. Azacitidine and venetoclax in previously untreated acute myeloid leukemia. N Engl J Med. 2020;383(7):617-629.

9. Short NJ, Kantarjian H. When less is more: reevaluating the role of intensive chemotherapy for older adults with acute myeloid leukemia in the modern era. $\mathrm{J}$ Clin Oncol. 2021;39(28):3104-3108.

10. Wei AH, Döhner H, Pocock C, et al. QUAZAR AML-001 Trial Investigators. Oral azacitidine maintenance therapy for acute myeloid leukemia in first remission. $N$ Engl $J$ Med. 2020;383(26):2526-2537.

11. Short NJ, Tallman MS, Pollyea DA, Ravandi F, Kantarjian H. Optimizing risk stratification in acute myeloid leukemia: dynamic models for a dynamic therapeutic landscape. J Clin Oncol. 2021;39(23):2535-2538. 